\title{
The Effect of Short Duration Electric Current on the Quasi-Static Tensile Behavior of Magnesium AZ31 Alloy
}

\author{
Trung Thien Nguyen, ${ }^{1}$ Thai Vinh Nguyen, ${ }^{1}$ Sung-Tae Hong, ${ }^{1}$ Moon-Jo Kim, \\ Heung Nam Han, ${ }^{2}$ and Fabrice Morestin ${ }^{3}$ \\ ${ }^{1}$ School of Mechanical Engineering, University of Ulsan, Nam-gu, Ulsan 680-749, Republic of Korea \\ ${ }^{2}$ Department of Materials Science \& Engineering and Center for Iron \& Steel Research, RIAM, Seoul National University, \\ Gwanak-gu, Seoul 151-742, Republic of Korea \\ ${ }^{3}$ Laboratory of Contact and Structure Mechanics, INSA-Lyon, 20, Avenue Albert Einstein, 69621 Villeurbanne, France
}

Correspondence should be addressed to Sung-Tae Hong; sthong@ulsan.ac.kr

Received 24 February 2016; Accepted 17 May 2016

Academic Editor: Pavel Lejcek

Copyright (C) 2016 Trung Thien Nguyen et al. This is an open access article distributed under the Creative Commons Attribution License, which permits unrestricted use, distribution, and reproduction in any medium, provided the original work is properly cited.

\begin{abstract}
The effect of a single pulse of electric current with short duration on the quasi-static tensile behavior of a magnesium AZ31 alloy is experimentally investigated. A single pulse of electric current with duration less than 1 second is applied to the specimen, while the specimen is being deformed in the plastic region under quasi-static tensile loads. After a nearly instant decrease of flow stress at the pulse of electric current, the flow stress shows strain hardening until the failure of the specimen. The experimental result shows that the strain-hardening parameters (the strength coefficient and the strain-hardening exponent) of the hardening curve after the electric current strongly depend on the applied electric energy density (electric energy per unit volume). Empirical expressions are suggested to describe the hardening behavior after the pulse as a function of the electric energy density and are compared with the empirical expressions suggested for advanced high-strength steels.
\end{abstract}

\section{Introduction}

Recent efforts in the automotive industries to improve fuel efficiency are reflected in the increasing use of lightweight materials. Magnesium alloys, which are common lightweight nonferrous alloys, provide a very high strength-to-weight ratio among commonly used structural metal alloys. However, the limited formability of magnesium alloys at room temperature hinders extensive use of those lightweight alloys in automotive industries.

The formability of magnesium alloys can be enhanced by forming the material at an elevated temperature. However, the forming at an elevated temperature may induce a few noticeable drawbacks, including increased adhesion between the specimen and die, reduced lubrication effectiveness, and decreased die strength [1]. Also, the elevated temperature forming can be time consuming and often requires a significant amount of initial capital investment. Therefore, forming methods that can enhance the formability of magnesium alloys without an excessive temperature increase or a high capital investment are still desired.

It has been argued that the material property of a metal can be altered by simply applying electricity to the metal during deformation (so-called electroplasticity) without a significant increase in temperature $[2,3]$. The presence of a continuous electric current during plastic deformation of a metal may reduce the flow stress of the metal significantly, as reported by Ross et al. [4] and Perkins et al. [5]. Under compression, the formability of a metal may be significantly enhanced with a continuous electric current [5]. However, as shown by Ross et al. [4], a continuously applied electric current may reduce the maximum achievable elongation of a metal under tension, probably due to the continuously decreasing cross-sectional area of the specimen during tension, which causes a continuous increase of the electric resistance of the specimen and consequently excessive heating in the gage section. 
Various electroplasticity theories have been proposed to explain the mechanical behavior during electrically assisted deformation [6-8]. The electroplasticity theories generally share an argument that the applied electric energy has effects, which are distinct from joule heating, on the dislocation in metals. However, it also needs to be noted that under certain mechanical, electrical, and thermal conditions the mechanical behavior associated with electrically assisted deformation can be modeled satisfactory using thermal-mechanical coupled models, without the need for electroplasticity theory [ 9 , 10]. In addition, for some cases, the effect of electric current on the mechanical behavior of metal alloys was observed to be insignificant $[9,11]$.

While a completely satisfactory theory to explain the mechanical behavior during electrically assisted deformation is still under investigation, studies have shown that the disadvantage of the reduced maximum elongation of metals under tension with a continuous electric current could be overcome by applying electric current periodically (a pulsed electric current) instead of continuously [12-15]. According to Roth et al. [12], elongation close to $400 \%$ of the specimen gage length was achieved by applying a pulsed electric current to 5754 aluminum alloy specimens under tension. Salandro et al. [1] also investigated the effect of pulse duration and current density on the tensile behavior of $\mathrm{Mg}$-AZ31BO specimens and showed that the elongation of the selected magnesium alloy could be significantly enhanced depending on the process parameters. More recently, Kim et al. [13] and Roh et al. [14] also showed that the formability of 5052 aluminum alloy specimens could be significantly enhanced by applying a pulsed electric current to the specimens during tension. In addition, Liu et al. [16] investigated the effect of pulsed electric current with high frequency on deformation mechanism of a magnesium AZ31B alloy during tension test. Their result showed that the electric current reduced the work hardening rate and flow stress, and the effect of electroplasticity depended on the Zener-Hollomon parameter.

In general, with a pulsed electric current, the tensile flow stress of a metal alloy shows a significant and nearly instant decrease when the electric current is applied to the specimen $[1,12-15]$. This nearly instant decrease in flow stress can be defined as the stress-drop [14]. Once the electric current is removed from the specimen, the stress rapidly increases and shows strain hardening until the next pulse of electric current (this can be defined as a local stress-strain curve [14]). The alternating of the stress-drop and the local stress-strain curve results in the tensile stress-strain curve under a pulsed electric current with a unique ratchet shape. It should be noted that, in the study of Kim et al. [13], microstructural observations confirmed that the mechanical behavior under a pulsed electric current may not be simply explained by the effect of resistance heating, and the electric current itself could play a distinct role, the electric current-induced annealing.

Unfortunately, experimental data and quantitative descriptions of the mechanical behavior of metals, especially magnesium alloys, under a pulsed electric current are still very limited. Also, it is naturally speculated that the mechanical behavior of a metal alloy under a single pulse of electric current should be fully investigated as a first step. In the present study, the mechanical behavior of magnesium AZ31 alloy under a single pulse of electric current is experimentally investigated.

\section{Material and Methods}

In the present study, magnesium $(\mathrm{Mg}) \mathrm{AZ31}$ alloy sheets with a thickness of $1 \mathrm{~mm}$ were used. For normal quasi-static tensile tests without electric current (baseline tests) and quasi-static tensile tests with a single pulse of electric current, typical tensile specimens with a $10 \mathrm{~mm}$ gage width and $50 \mathrm{~mm}$ gage length were fabricated along the rolling direction of the sheet according to ASTM-E08.

The quasi-static tensile tests were conducted using a universal testing machine with a constant displacement rate of $2 \mathrm{~mm} / \mathrm{min}$ (corresponding strain rate of $0.05 / \mathrm{min}$ ) at room temperature. The force history during the experiment was measured by a CSDH load cell (Bongshin, South Korea) with a maximum capacity of $300 \mathrm{kN}$ as a function of displacement using a PC-based data acquisition system. The displacement history was measured using a LX500 laser extensometer (MTS, USA) by attaching tape markers to the specimen to fix the gage length to $50 \mathrm{~mm}$. The displacement of the gage length was then simply converted to the corresponding engineering strain. A digital image correlation (DIC) system, named ICASOFT, was additionally employed in a few experiments by applying a speckle pattern with a black paint on one side of the gage section to confirm the strain history via laser extensometer.

For the quasi-static tensile test with a single pulse of electric current, the electric current was generated by a Vadal SP-1000U welder (Hyosung, South Korea) and applied to the specimen during tensile deformation, as schematically shown in Figure 1(a). Note that the electric current was applied to the specimen over a given duration at the selected engineering strain without stopping the tensile displacement, as schematically shown in Figure 1(b). A set of bakelite insulators was inserted between the specimen and each grip to isolate the electricity from the testing equipment. The thermal effect due to the applied electric energy, that is, the resistive heating of the specimen, was evaluated by measuring the temperature profile of the specimen during the test. A FLIRT-T621 infrared thermal imaging camera (FLIR, Sweden) was employed to continuously monitor the temperature profile of the specimen. High temperature black paint was applied to the side facing the thermal imaging camera to stabilize the emissivity of the specimen.

Electric pulse parameters were selected for the experiment as listed in Table 1. Four different sets of electric pulse parameters were employed by combining four different electric current densities based on the original cross-sectional area of the specimen (nominal electric current density $\rho_{i_{-o}}$ ) with a fixed duration of electric current (pulse duration $t_{d}$ ) of $0.5 \mathrm{sec}$ and a fixed displacement of $1.5 \mathrm{~mm}$ (corresponding engineering strain of 3\%) at the moment of electric current.

According to Roh et al. [14], the electroplastic behavior of metal under a pulsed electric current may be described using the applied electric energy per unit volume (electric energy density), which is a function of the electric current 


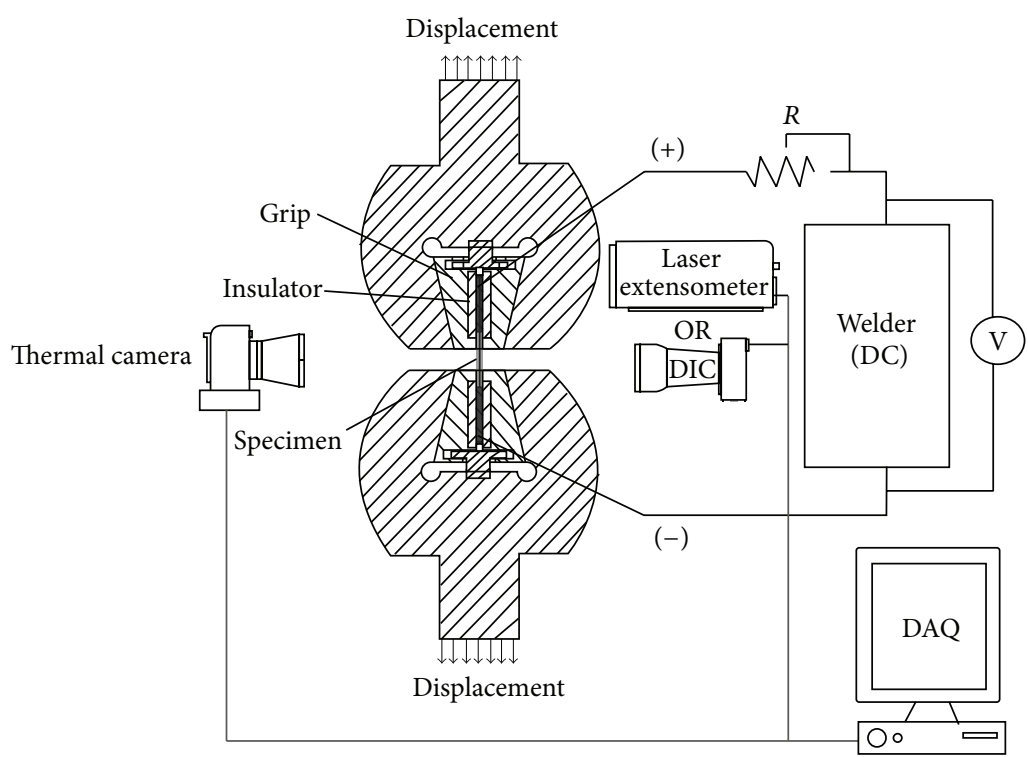

(a)

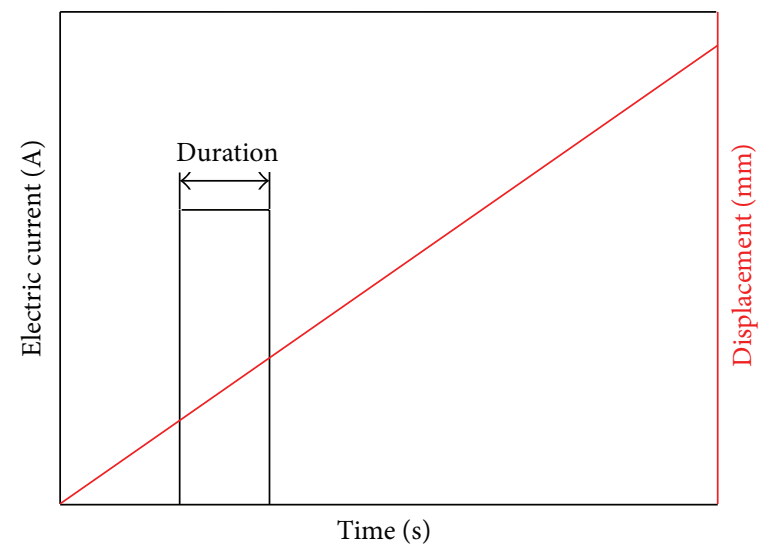

(b)

FIGURE 1: Schematics of (a) the experimental set-up and (b) applying a single pulse of electric current during deformation.

density and the pulse duration. The electric energy density may be defined based on the original volume of the gage section of the specimen (nominal electric energy density $j_{o}$ ) or the volume of the gage section at the moment of electric current (true electric energy density $j[14]$ ). The nominal electric energy density based on the original volume of the specimen, $V_{o}$, can be simply written as

$$
j_{o}=\frac{I^{2} R_{o} t_{d}}{V_{o}}
$$

where $I$ and $R_{o}$ represent the electric current (A) and the electric resistance of the undeformed specimen $(\Omega)$, respectively.

The true electric energy density also can be calculated in the same manner using the volume and electric resistance at the moment of electric current. The true electric energy density then can be simply related to the nominal electric energy density [14]. The calculation is quite straightforward and is not presented here. Note that since the engineering strain at the moment of electric current is fixed through all experiments, the same nominal electric energy density means the same true electric energy density. In the present study, the nominal electric energy density is used to discuss the mechanical behavior of the selected alloy under a single pulse of electric current. For calculation of electric energy, the electric resistance $R_{o}$ of the specimen was measured by a four-point measurement system, which applies a constant electrical current to the specimen and measures the resulting voltage drop by a separate set of leads [17].

In addition to the tensile test with a single pulse of electric current, reloading tensile tests after a single pulse of electric current were also conducted using the parameter sets in Table 1. In the reloading test, a single pulse of electric current was applied to the specimen at the engineering strain of $3 \%$ and the test was manually stopped immediately after the duration of the electric current. The specimen was removed from the universal testing machine and then 
TABLE 1: Electric pulse parameters.

\begin{tabular}{lcc}
\hline $\begin{array}{l}\text { Current density } \\
\left(\mathrm{A} / \mathrm{mm}^{2}\right)\end{array}$ & $\begin{array}{c}\text { Pulse duration } \\
(\mathrm{sec})\end{array}$ & $\begin{array}{c}\text { Engineering strain at the } \\
\text { electric current }(\%)\end{array}$ \\
\hline 30 & & \\
45 & 0.5 & 3 \\
60 & & \\
75 & & \\
\hline
\end{tabular}

${ }^{*}$ Four different sets of electric pulse parameters by combining four different electric current densities with a fixed duration of electric current.

remounted and reloaded until fracture. A baseline reloading test was also conducted in the same manner without applying electric current. At least three specimens were tested for each parameter set to verify the repeatability of the result.

Finally, the effect of electric current during plastic deformation on the microstructure of the selected magnesium alloy was briefly evaluated by electron back-scatter diffraction (EBSD) analysis using a filed emission gun scanning electron microscope (Hitachi, Japan) equipped with an EDAX-TSL EBSD system and OIM 5.2 software. For microstructural analysis, tensile tests with the set of electric pulse parameters $\rho_{i \_o}=75 \mathrm{~A} / \mathrm{mm}^{2}$ and $t_{d}=0.5 \mathrm{sec}$, which results in the highest electric energy density among the parameter sets, were selected. Deformation was manually stopped immediately after the duration of the electric current at the engineering strain of 3\%, and the specimen was removed from the test. A baseline tensile test was also manually stopped at the engineering strain of $3 \%$ and the specimen was removed from the test for comparison. The samples for EBSD analysis were prepared perpendicular to the tensile direction of the specimens. The samples were electrolytically polished in a $100 \mathrm{~mL}$ perchloric acid $+900 \mathrm{~mL}$ methanol solution using a Lectropol-5 electrolytic polisher (Struers, Denmark) after standard metallographic grinding. In EBSD analysis, the accelerating voltage and working distance were $15 \mathrm{kV}$ and $15 \mathrm{~mm}$, respectively.

\section{Results and Discussion}

The repeatability of the experimental result was quite good for all the parameter sets selected, as shown by the results with $\rho_{i_{-}}=60 \mathrm{~A} / \mathrm{mm}^{2}$ and $t_{d}=0.5 \mathrm{sec}$ in Figure 2. The engineering stress-strain curves of the selected magnesium alloy from the quasi-static tensile tests with a single pulse of electric current showed a nearly instant and significant decrease of flow stress, the stress-drop [14, 18], when the electric current was applied to the specimen. Once the electric current was removed from the specimen, the flow stress of the selected magnesium alloy rapidly increased again and showed a strain-hardening behavior until fracture. This trend of nearly instant stress-drop and subsequent strain-hardening behavior under a single pulse of electric current corresponds well with the experimental results of advanced high-strength steel under similar conditions [18].

The maximum temperature of the specimen in the duration of electric current was only $158^{\circ} \mathrm{C}$ even with the electric pulse parameters, $\rho_{i_{-}}=75 \mathrm{~A} / \mathrm{mm}^{2}$ and $t_{d}=0.5 \mathrm{sec}$, which

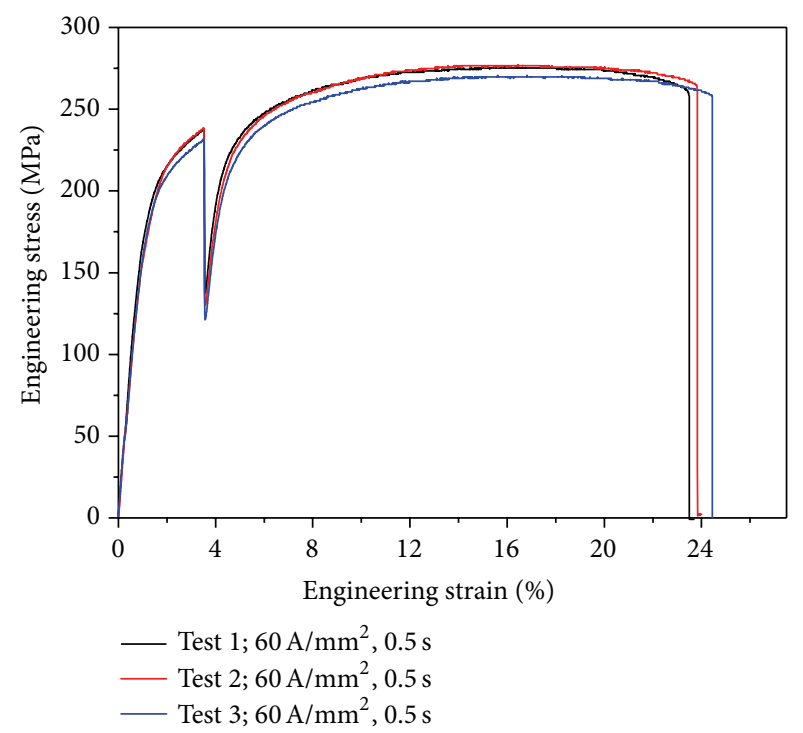

FIGURE 2: The repeatability of experimental result: $\rho_{i_{-}}=60 \mathrm{~A} / \mathrm{mm}^{2}$, $t_{d}=0.5 \mathrm{sec}$.

induced the maximum electric energy density among the electric pulse parameter sets selected in the present study, as shown in the temperature profile along the center-line of the gage of specimen (Figure 3(a)). Note that the temperature of $158^{\circ} \mathrm{C}$ is still below the hot working temperature range for the magnesium alloy [19]. Also, it should be noted that once the electric current was removed from the specimen, the temperature of the specimen rapidly decreased and most of the deformation occurred near room temperature, as shown in Figure 3(b). Therefore, as previously reported [5, 12-15, 18, 20], the thermal effect of applied electric current may not be a major reason for the mechanical behavior of the selected magnesium alloy under a single pulse of electric current, at least with the electric pulse parameter sets used in the present study.

For the given range of electric energy density, the magnitude of the stress-drop increased as the electric energy density increased, as shown in Figure 4. The increase of stress-drop as a function of the electric energy density may be linearly fitted, as shown in Figure 5. The percent magnitude of stress-drop in Figure 5 was simply calculated by dividing the magnitude of the stress-drop at the moment of electric pulse by the stress immediately before applying the electric current. Regarding the mechanism of the stress-drop, we suspect that the electric current-induced annealing [13] and the thermal effect by joule heating could be involved, while some effect of thermal expansion may also be considered [18]. Further research is required to clearly explain the stress-drop mechanism.

Also, a slight increase of the elongation at fracture was observed for the selected magnesium alloy, especially with the electric energy densities higher than $0.084 \mathrm{~J} / \mathrm{mm}^{3}$, while the ultimate tensile strength (UTS) after electric current slightly decreased, as shown in Figures 6(a) and 6(b). This result may suggest that the electric current-induced annealing occurred in the selected magnesium alloy [13]. Note that the ductility of advanced high-strength steel was severely decreased by a 


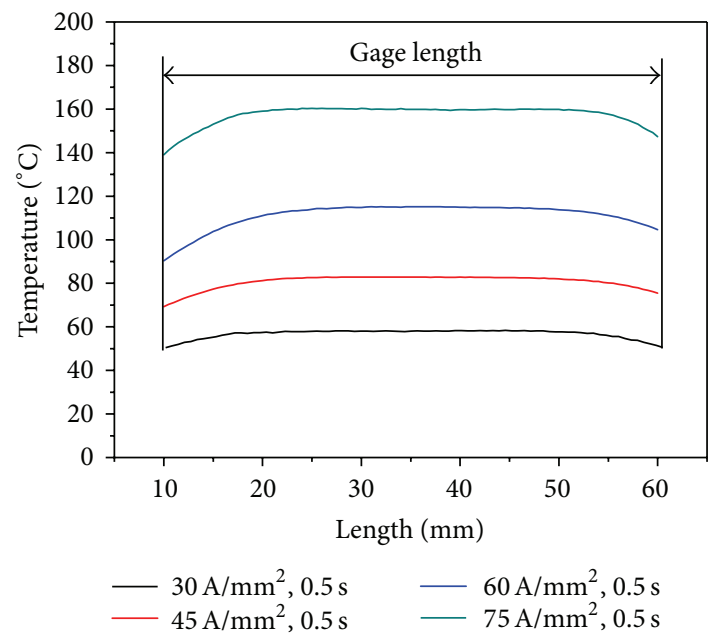

(a)

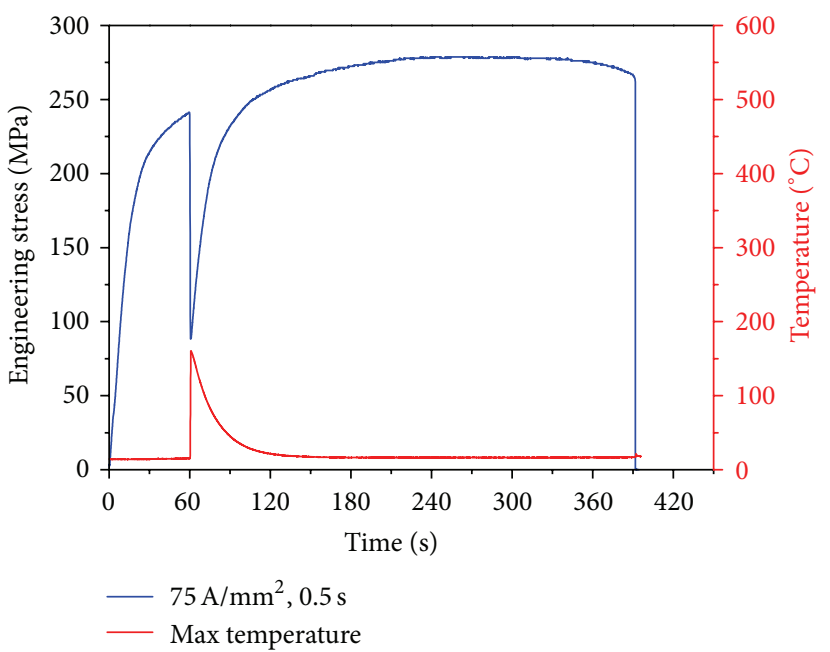

(b)

FIgURE 3: (a) Temperature profiles along the center-line of the gage of specimen and (b) a history of the maximum temperature within the gage of specimen during a tensile test with $\rho_{i_{-}}=75 \mathrm{~A} / \mathrm{mm}^{2}, t_{d}=0.5 \mathrm{sec}$.

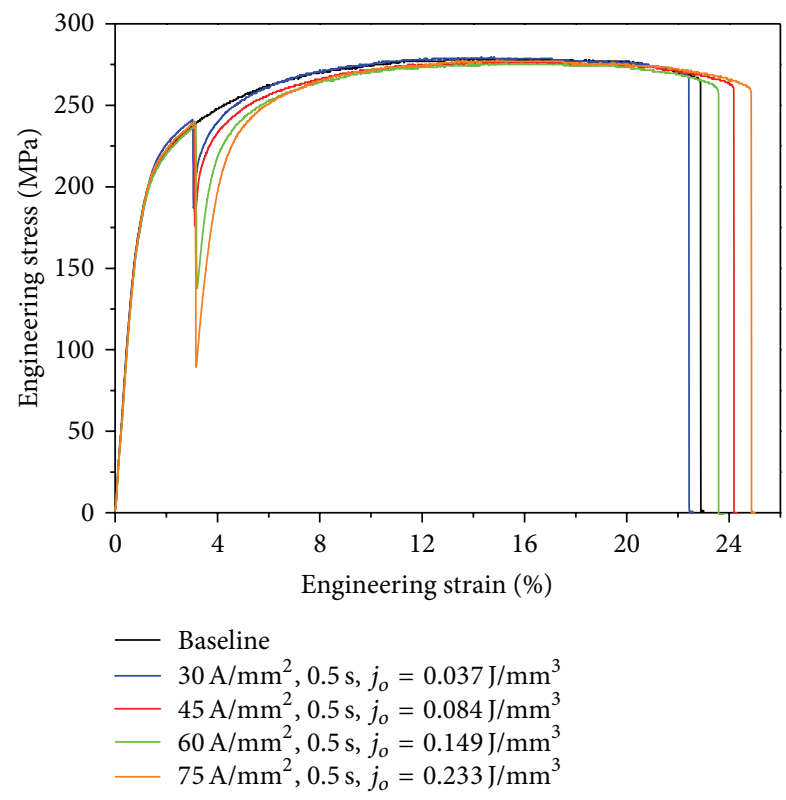

FIGURE 4: Engineering stress-strain curves with different electric pulse parameters.

single pulse of electric current during deformation [18]. The different mechanical responses of the magnesium alloy in the present study and the advanced high-strength steel in Kim et al. [18] to the single pulse of electric current strongly suggest that the effect of electric current during deformation on the mechanical behavior of a metal alloy depends on the selected metal alloy, as well as the electric pulse parameters.

One interesting aspect regarding the effect of the single pulse of electric current on the mechanical behavior of the selected alloy is that diffuse necking intensified with the single pulse of electric current in the plastic region. As shown in Figure 7, even with $\rho_{i_{-o}}=30 \mathrm{~A} / \mathrm{mm}^{2}$ and $t_{d}=0.5 \mathrm{sec}$, which

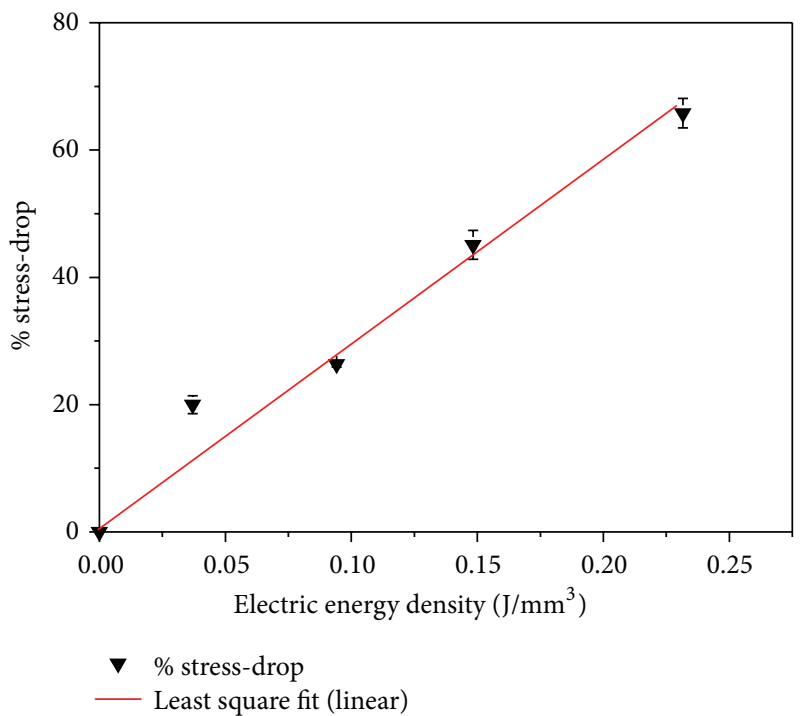

FIGURE 5: The percent magnitude of stress-drop as a function of the electric energy density (the least square fit).

induce the lowest electric energy density among the electric pulse parameter sets selected in the present study, diffuse necking became quite pronounced in comparison with the result of the baseline test (no electric current). Note that, for $\rho_{i \_o}=30 \mathrm{~A} / \mathrm{mm}^{2}$ and $t_{d}=0.5 \mathrm{sec}$, the temperature of the specimen was approximately $50^{\circ} \mathrm{C}$ even in the duration of electric current as shown in Figure 3(a), and most of the deformation after the electric current occurred near room temperature. The pronounced diffuse necking with a single pulse of electric current may suggest that the electric current during deformation induced the mechanical behavior at an elevated temperature in conventional hot or warm forming, while the increase of temperature was much smaller. 


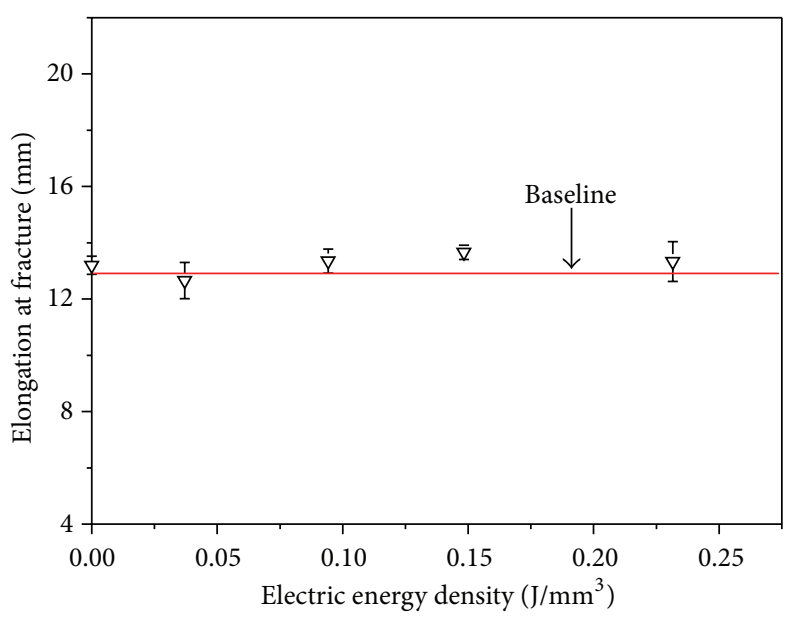

$\nabla$ Elongation at fracture

(a)

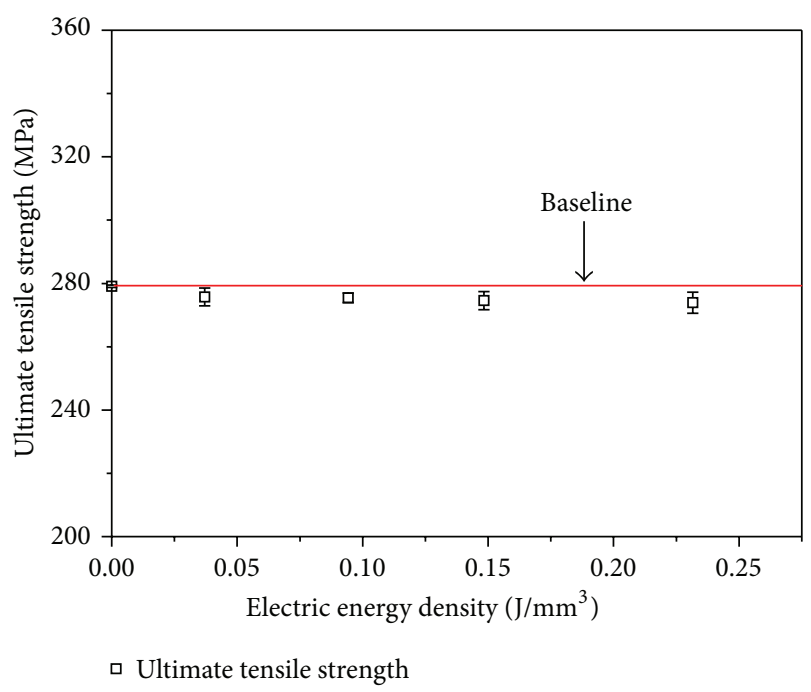

(b)

FIGURE 6: (a) The elongation at fracture and (b) the UTS as functions of the electric energy density.

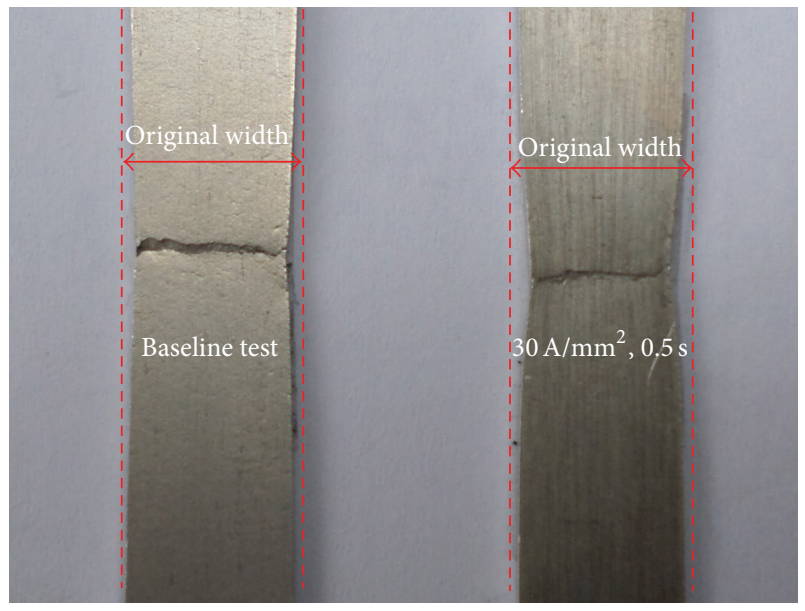

Figure 7: Intensified diffuse necking with a single pulse of electric current in plastic region. Note that the electric parameter set, $\rho_{i_{-} o}=$ $30 \mathrm{~A} / \mathrm{mm}^{2}$ and $t_{d}=0.5 \mathrm{sec}$, induces the lowest electric energy density among the electric pulse parameter sets selected in the present study.

The hardening behavior after a single pulse of electric current, or after the stress-drop, is quite important in many aspects, for example, design of a forming process utilizing the effect of electric current during deformation. The electric energy density applied to the specimen in plastic region induced a stress-drop and altered the hardening behavior of the magnesium alloy, after the electric current was removed (Figure 4). For the analysis of the hardening behavior after the electric current, the engineering stress-strain curve was first converted to a true stress-strain curve up to necking initiation, as shown in Figure 8. The true stress after the electric current was then fitted as a function of the true strain

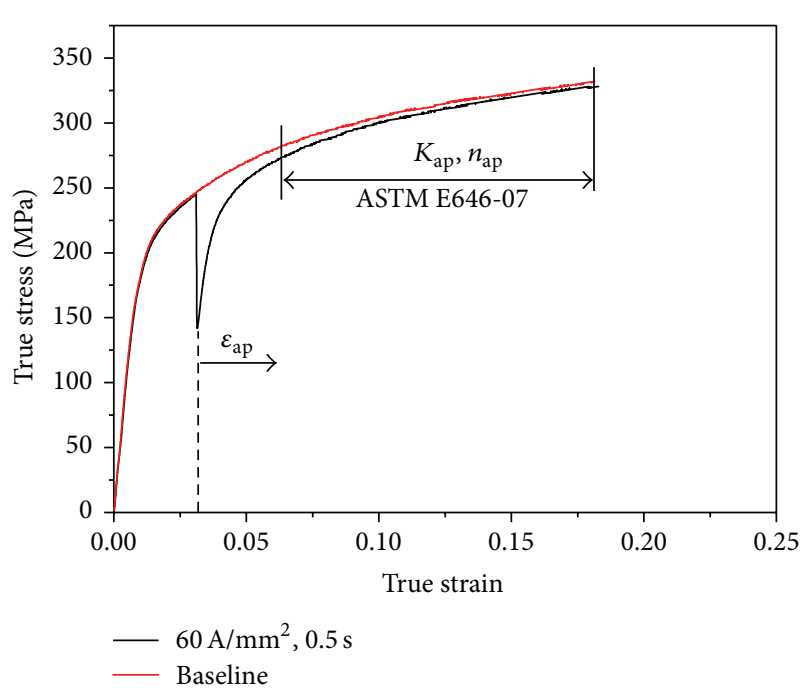

FIGURE 8: A definition of the true strain $\varepsilon_{\text {ap }}$ and the calculation of hardening parameters after the pulse of electric current.

$\varepsilon_{\text {ap }}$ after the electric current $\left(\varepsilon_{\text {ap }}=0\right.$ at the moment of electric current) using the power law expression:

$$
\sigma=K_{\mathrm{ap}}\left(\varepsilon_{\mathrm{ap}}\right)^{n_{\mathrm{ap}}},
$$

where $K_{\mathrm{ap}}$ and $n_{\mathrm{ap}}$ represent the strength coefficient and the strain-hardening exponent after the electric current, respectively. The subscript ap denotes "after the pulse of electric current" or simply "after the electric current." Note that, for the simplicity of the study, it is assumed that $\sigma=0$ at $\varepsilon_{\text {ap }}=0$ in (2). However, the simple equation suggested in (2) still describes the shape of the true stress-strain curve after the single pulse of electric current reasonable well in large deformation region $\left(\varepsilon_{\text {ap }} \gg 0\right)$, as presented below. 


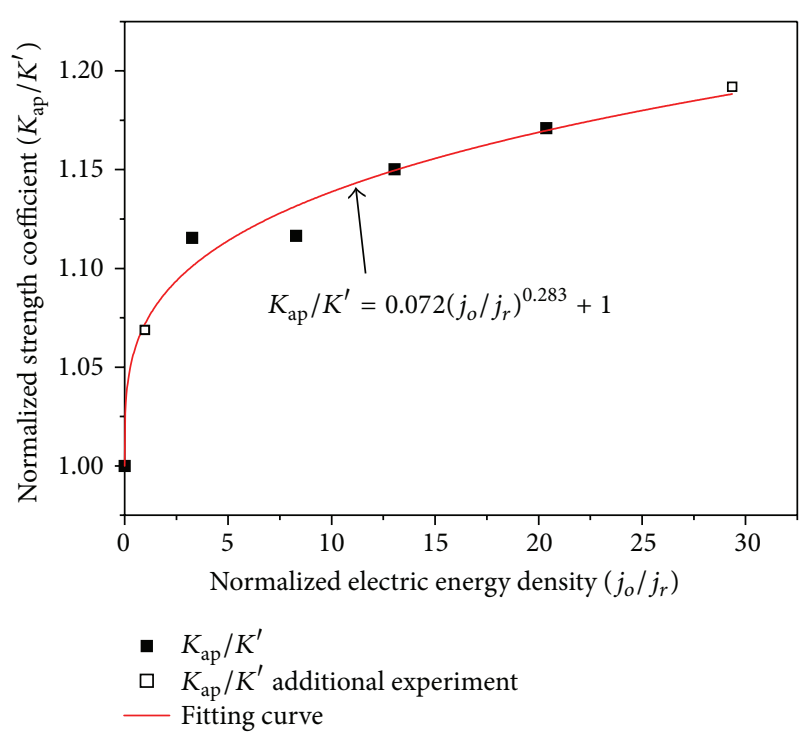

(a)

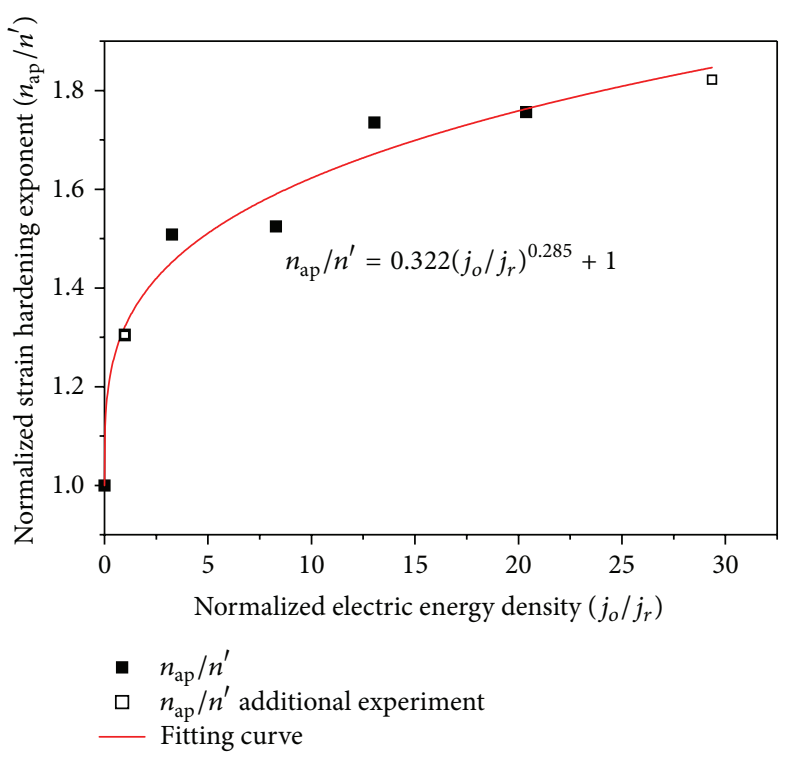

(b)

FIGURE 9: The normalized hardening parameters as functions of the electric energy density: (a) $K_{\text {ap }} / K^{\prime}$ and (b) $n_{\text {ap }} / n^{\prime}$. Note that the results of additional experiments were plotted and after that the fitted curves were plotted.

The hardening parameters $K_{\mathrm{ap}}$ and $n_{\mathrm{ap}}$ were calculated from the true stress-strain curve after the electric current according to ASTM E646-07. The hardening parameters $K_{\text {ap }}$ and $n_{\text {ap }}$ after the electric current were then normalized by $K^{\prime}$ and $n^{\prime}$, respectively, which were calculated from the part of the baseline true stress-strain curve after the engineering strain of 3\%, as shown in Figures 9(a) and 9(b). Note that $K^{\prime}$ and $n^{\prime}$ correspond to $K_{\mathrm{ap}}$ and $n_{\mathrm{ap}}$ at $j_{o}=0$, respectively. As shown in Figures 9(a) and 9(b), both $K_{\text {ap }}$ and $n_{\text {ap }}$ increased as the applied electric energy density increased, which suggests that the applied electric current induced an annealing effect [13] in the selected magnesium alloy. The normalized hardening parameters $K_{\mathrm{ap}} / K^{\prime}$ and $n_{\mathrm{ap}} / n^{\prime}$ as functions of the normalized electric energy density $j_{o} / j_{r}$ could be simply fitted:

$$
\begin{aligned}
& \frac{K_{\mathrm{ap}}}{K^{\prime}}=0.072\left(\frac{j_{o}}{j_{r}}\right)^{0.283}+1 \\
& \frac{n_{\mathrm{ap}}}{n^{\prime}}=0.322\left(\frac{j_{o}}{j_{r}}\right)^{0.285}+1,
\end{aligned}
$$

where $j_{r}$ is the reference electric energy density determined as the threshold electric energy density corresponding to the stress-drop of $2 \%$. The effectiveness of the suggested empirical expressions in (3) is confirmed by comparing the fitted curves and the results of additional experiments with two different electric energy densities (hollow symbols in Figures 9(a) and 9(b)). Note that the results of additional experiments were plotted and after that the fitted curves were plotted.

It is interesting to compare the result of the present study with that of advanced high-strength steel with similar experimental parameters [18]. According to Kim et al. [18], the hardening parameters after the pulse of electric current for the advanced high-strength steel also increased as the electric energy density increased. Note that, in the study of Kim et al. [18], the normalized hardening parameters were plotted as functions of true electric energy density. Replotting the results of Kim et al. [18] as functions of the nominal electric energy density would not change the general trend of the fitted curves. For both of the normalized hardening parameters $K_{\mathrm{ap}} / K^{\prime}$ and $n_{\mathrm{ap}} / n^{\prime}$, the slopes of the fitted curves for the selected magnesium alloy decrease as the electric energy density increases, as shown in Figures 9(a) and 9(b), while those for the advanced high-strength steel in Kim et al. [18] increase as the electric energy density increases. As a result, the trends of the normalized hardening parameters for the selected magnesium alloy were fitted by the power law with exponents less than 1 to describe the decreasing slopes with increasing electric energy density for both $K_{\text {ap }} / K^{\prime}$ and $n_{\text {ap }} / n^{\prime}$. However, the exponents of the fitted curves for the advanced high-strength steel [18] were greater than 1 (increasing slopes with increasing electric energy density) for both $K_{\mathrm{ap}} / K^{\prime}$ and $n_{\mathrm{ap}} / n^{\prime}$. The comparison of the present study and the result for the advanced high-strength steel [18] strongly suggests that the mechanical behavior after the electric current during plastic deformation depends on the material composition, as well as the electric pulse parameters.

The result of the reloading tensile tests in Figure 10 shows that the single pulse of electric current decreased the yield stress in the reloading curve at room temperature, which confirms that the electric current-induced annealing [13] has occurred in the selected magnesium alloy. The reloading tensile tests also reveal one interesting effect of electric current on the transition from the elastic region to the plastic region. As shown in the insert in Figure 10, a slight bump 


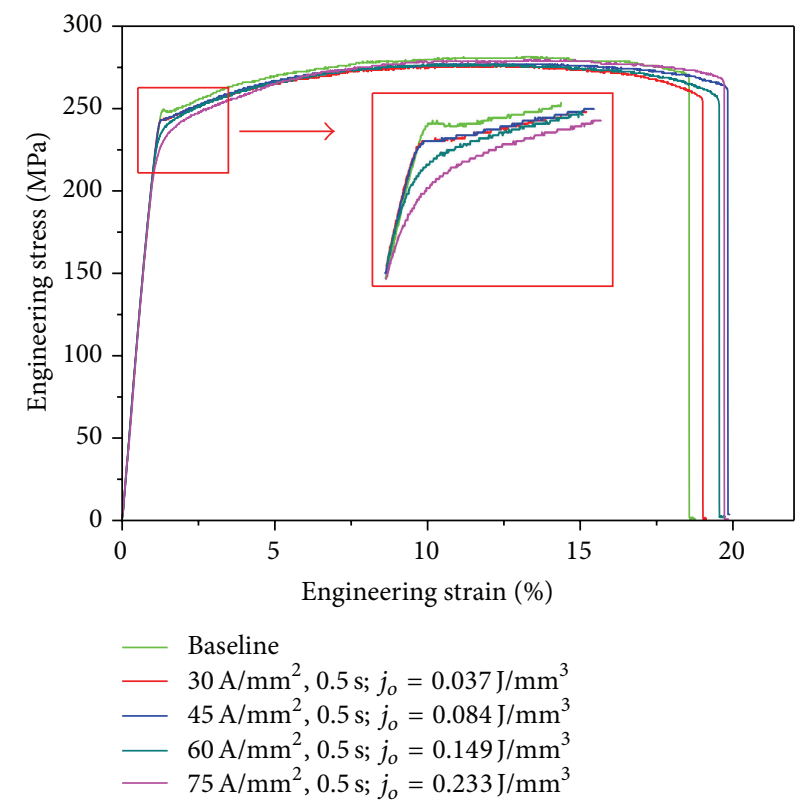

FIGURE 10: The stress-strain curves of the reloading tensile tests.
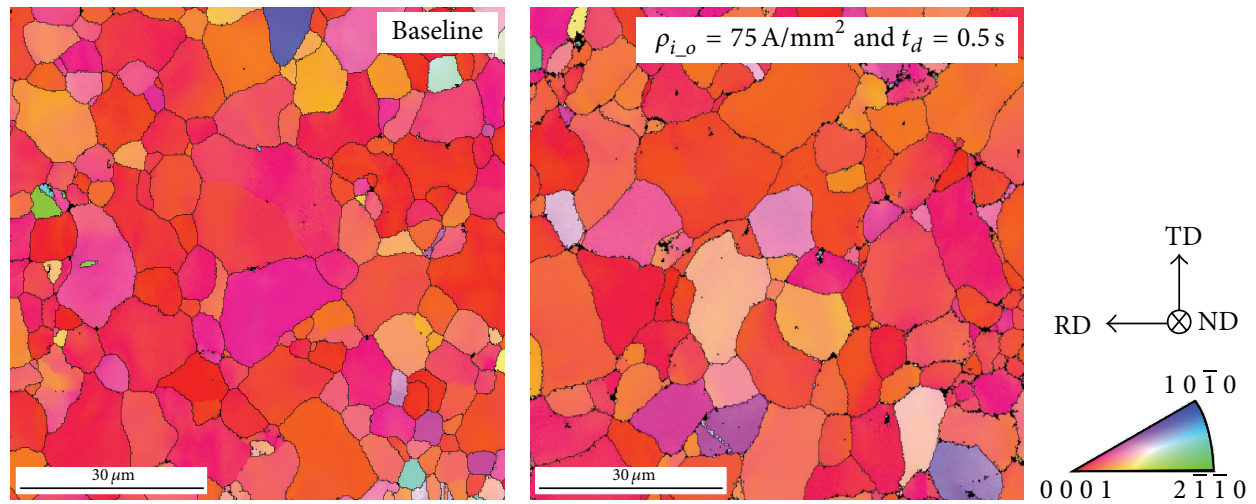

FIGURE 11: EBSD orientation (normal direction, ND) map with the electric pulse parameter set resulting in the highest electric energy in comparison with that without electric current (baseline).

occurred during transition from the elastic region to the plastic region in the baseline reloading curve. However, the bump became insignificant as the electric energy density increased and completely disappeared for the electric energy densities 0.149 and $0.233 \mathrm{~J} / \mathrm{mm}^{3}$. The result of the reloading tests at room temperature once again confirms that the mechanical behavior under a single pulse of electric current during plastic deformation is not simply a result of joule heating. Further discussion regarding the reloading behavior after the electric current is beyond the scope of the present study and will be conducted as a future work.

EBSD orientation (normal direction, ND) map of the specimen with the electric pulse parameter set resulting in the highest electric energy density among the parameter sets was compared with that of the specimen without electric current. The grain sizes of specimen without and with electric current were similar, $10.5 \pm 3.7 \mu \mathrm{m}$ and $11 \pm 4.2 \mu \mathrm{m}$, respectively, with a critical misorientation angle of $5^{\circ}$. Also, recrystallization was not observed by applying a single pulse of electric current, as shown in Figure 11. This seems reasonable since the maximum temperature during the test with $\rho_{i \_o}=75 \mathrm{~A} / \mathrm{mm}^{2}$ and $t_{d}=0.5 \mathrm{sec}$ was only $\sim 160^{\circ} \mathrm{C}$, which is significantly lower than the generally known temperature range, $250^{\circ} \mathrm{C} \sim 400^{\circ} \mathrm{C}$, required for recrystallization of magnesium AZ31 alloy [2123]. The result of EBSD analysis confirms that no significant microstructural change occurred for the selected magnesium alloy upon application of a single pulse of electric current during plastic tensile deformation.

\section{Conclusion}

The effect of a single pulse of electric current with short duration on the quasi-static tensile behavior of a magnesium AZ31 alloy was experimentally investigated. A nearly instant decrease of flow stress occurred upon a pulse of electric current and the flow stress showed strain hardening 
until the failure of the specimen. Power law expressions were suggested for the strain-hardening parameters after the electric current as functions of the electric energy density. While the suggested power law expressions are purely empirical, they were confirmed to effectively describe the trend of hardening parameters as a function of the electric energy density. One advantage of adopting the power law in describing the trend of hardening parameters is that the same equation form can be used to describe somewhat different trends for different materials by properly selecting the exponent of the power law. The result of the present study would be helpful in developing various electrically assisted manufacturing processes. However, it should be noted that the effects of strain rate and amount of plastic strain prior to the electric current were not considered. Also identifying the thermal and electroplastic effects on the stress-drop would be meaningful. Extensive future studies will be conducted to investigate those effects and consequently develop a more general empirical expression to describe the electroplastic behavior of various metal alloys.

\section{Competing Interests}

The authors declare that they have no competing interests.

\section{Acknowledgments}

This research was financially supported by the Ministry of Trade, Industry and Energy (MOTIE) and Korea Institute for Advancement of Technology (KIAT) through the Promoting Regional Specialized Industry. This research was also financially supported by the Ministry of Education (MOE) and National Research Foundation of Korea (NRF) grant funded by the Ministry of Science, ICT \& Future Planning (MSIP) (no. NRF-2015R1A5A1037627). Heung Nam Han and MoonJo Kim were supported by Technology Innovation Industrial Program funded by the Ministry of Trade, Industry and Energy, Republic of Korea (Grant no. 10052779, Development of Car Body Modularization Technology using Advanced Cold Forming and Welding Technologies of Low Density GIGA Grade Light Steel Sheets).

\section{References}

[1] W. A. Salandro, K. Ashraf, and J. T. Roth, “Tensile formability enhancement of magnesium AZ31B-0 alloy using electrical pulsing," Transactions of the North American Manufacturing Research Institute of SME, vol. 37, pp. 387-394, 2009.

[2] O. A. Troitskii, "Electromechanical effect in metals," ZhETF Pisma Redaktsiiu, vol. 10, pp. 18-22, 1969.

[3] H. Conrad, "Electroplasticity in metals and ceramics," Materials Science and Engineering A, vol. 287, no. 2, pp. 276-287, 2000.

[4] C. D. Ross, D. B. Irvin, and J. T. Roth, "Manufacturing aspects relating to the effects of direct current on the tensile properties of metals," Journal of Engineering Materials and Technology, vol. 129, no. 2, pp. 342-347, 2007.

[5] T. A. Perkins, T. J. Kronenberger, and J. T. Roth, "Metallic forging using electrical flow as an alternative to warm/hot working," Journal of Manufacturing Science and Engineering, vol. 129, no. 1, pp. 84-94, 2007.
[6] Y. Li, H. Chen, Y. Gu, and X. Hu, "Effect of electric current pulse on superplasticity of aluminium alloy 7475," Transactions of Nonferrous Metals Society of China (English Edition), vol. 6, no. 1, pp. 77-84, 1996.

[7] H. Conrad, "Effects of electric current on solid state phase transformations in metals," Materials Science and Engineering: A, vol. 287, no. 2, pp. 227-237, 2000.

[8] D. Roylance, Mechanical Properties of Materials, MIT Press, 2008.

[9] J. Magargee, F. Morestin, and J. Cao, "Characterization of flow stress for commercially pure titanium subjected to electricallyassisted deformation," Journal of Engineering Materials and Technology, vol. 135, no. 4, Article ID 041003, 2013.

[10] J. Magargee, R. Fan, and J. Cao, "Analysis and observations of current density sensitivity and thermally activated mechanical behavior in electrically-assisted deformation," Journal of Manufacturing Science and Engineering, vol. 135, no. 6, Article ID 061022, 2013.

[11] B. Kinsey, G. Cullen, A. Jordan, and S. Mates, "Investigation of electroplastic effect at high deformation rates for 304SS and Ti6Al-4V," CIRP Annals-Manufacturing Technology, vol. 62, no. 1, pp. 279-282, 2013.

[12] J. T. Roth, I. Loker, D. Mauck, M. Warner, S. F. Golovashchenko, and A. Krause, "Enhanced formability of 5754 aluminum sheet metal using electric pulsing," Transaction of NAMRI/SME, vol. 36, pp. 405-412, 2008.

[13] M.-J. Kim, K. Lee, K. H. Oh et al., "Electric current-induced annealing during uniaxial tension of aluminum alloy," Scripta Materialia, vol. 75, pp. 58-61, 2014.

[14] J.-H. Roh, J.-J. Seo, S.-T. Hong, M.-J. Kim, H. N. Han, and J. T. Roth, "The mechanical behavior of 5052-H32 aluminum alloys under a pulsed electric current," International Journal of Plasticity, vol. 58, pp. 84-99, 2014.

[15] W. A. Salandro, J. J. Jones, T. A. McNeal, J. T. Roth, S.-T. Hong, and M. T. Smith, "Formability of Al 5xxx sheet metals using pulsed current for various heat treatments," Journal of Manufacturing Science and Engineering, vol. 132, no. 5, Article ID 051016, 2010.

[16] K. Liu, X. Dong, H. Xie, Y. Wu, and F. Peng, "Influence of pulse current on deformation mechanism of AZ31B sheet during tension," Journal of Alloy and Compounds, vol. 676, pp. 106-112, 2016.

[17] S.-T. Hong and K. S. Weil, "Niobium-clad 304L stainless steel PEMFC bipolar plate material: tensile and bend properties," Journal of Power Sources, vol. 168, no. 2, pp. 408-417, 2007.

[18] M.-S. Kim, N. T. Vinh, H.-H. Yu et al., "Effect of electric current density on the mechanical property of advanced high strength steels under quasi-static tensile loads," International Journal of Precision Engineering and Manufacturing, vol. 15, no. 6, pp. 1207-1213, 2014.

[19] V. Tabacaru and N. Oancea, "Sheet metal forming of magnesium wrought alloys-formability and process technology," Journal of Materials Processing Technology, vol. 115, no. 1, pp. 1419, 2001.

[20] C. R. Green, T. A. McNeal, and J. T. Roth, "Springback elimination for Al-6111 alloys using Electrically-Assisted Manufacturing (EAM)," Transaction of the North American Manufacturing Research Institute of SME, vol. 37, pp. 403-410, 2009.

[21] J. Liu, Z. Cui, and C. Li, "Modelling of flow stress characterizing dynamic recrystallization for magnesium alloy AZ31B," Computational Materials Science, vol. 41, no. 3, pp. 375-382, 2008. 
[22] G. Bajargan, G. Singh, D. Sivakumar, and U. Ramamurty, "Effect of temperature and strain rate on the deformation behavior and microstructure of a homogenized AZ31 magnesium alloy," Materials Science and Engineering: A, vol. 579, pp. 26-34, 2013.

[23] M. Wang, B. Y. Zong, and G. Wang, "Grain growth in AZ31 Mg alloy during recrystallization at different temperatures by phase field simulation," Computational Materials Science, vol. 45, no. 2, pp. 217-222, 2009. 

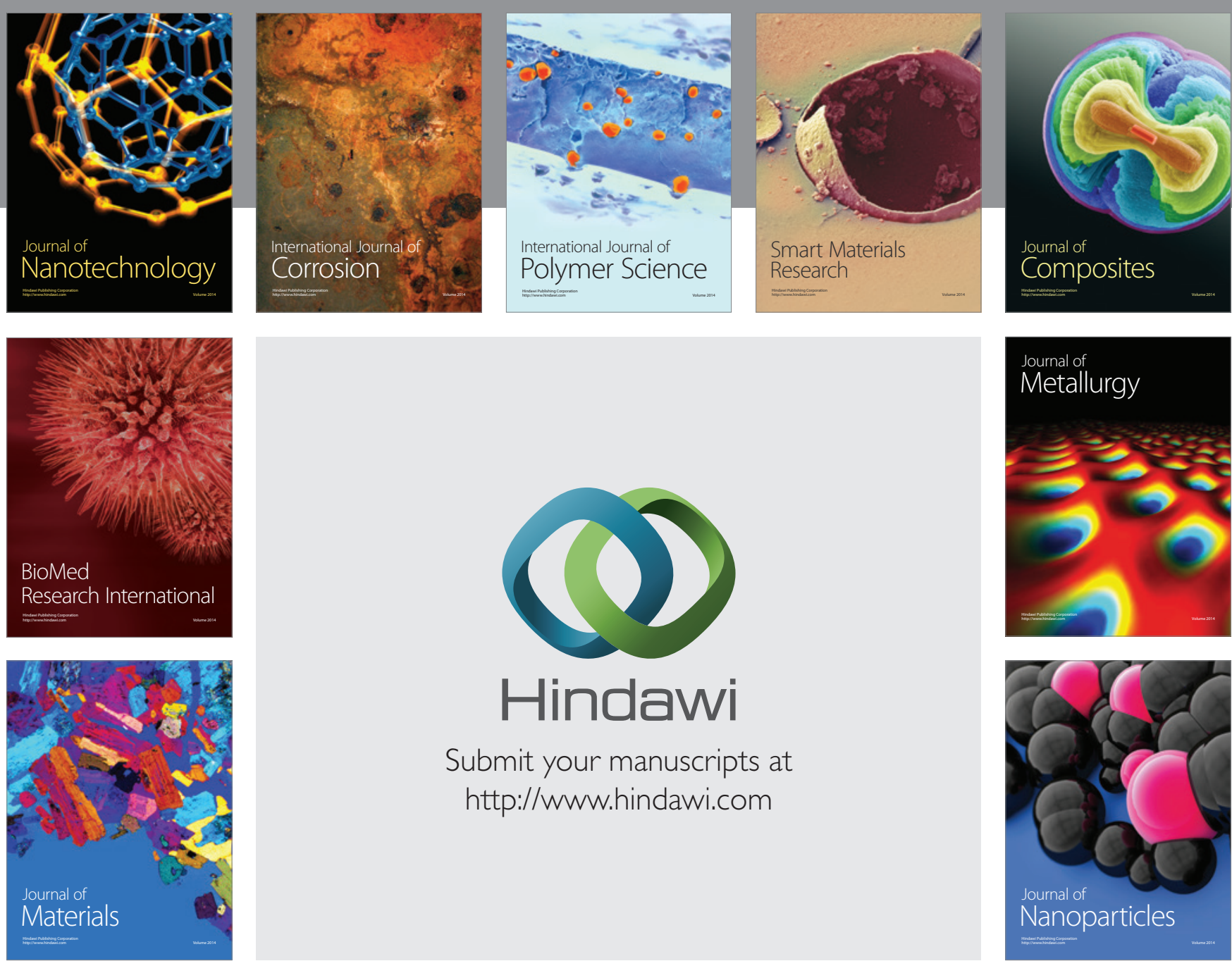

\section{Hindawi}

Submit your manuscripts at

http://www.hindawi.com

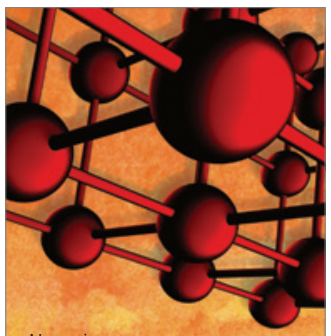

Materials Science and Engineering
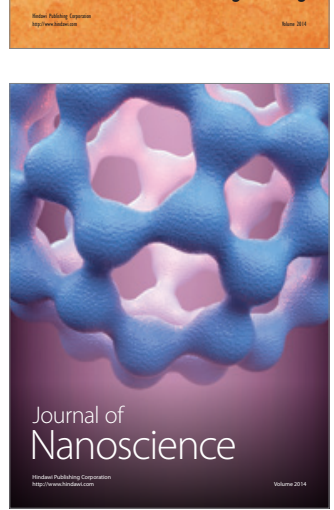
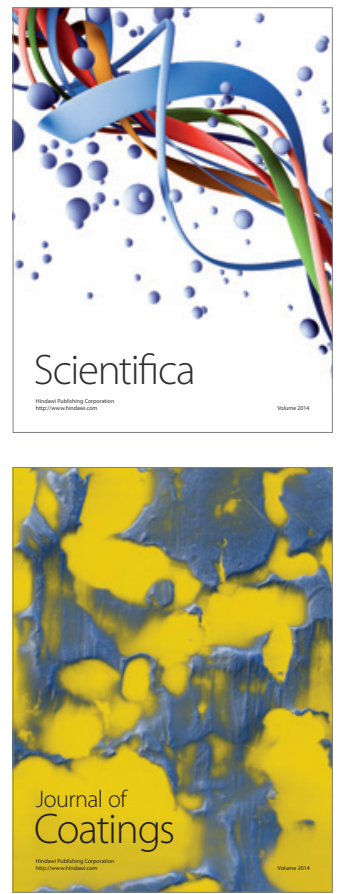
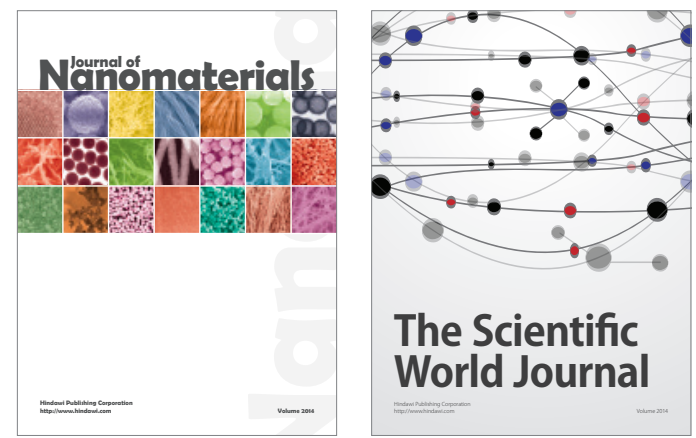

The Scientific World Journal
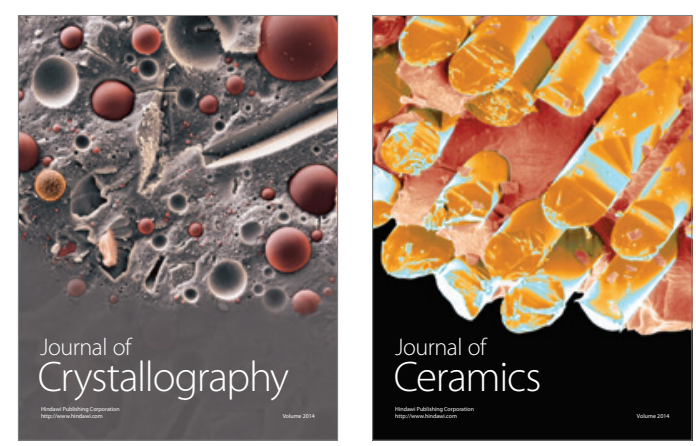
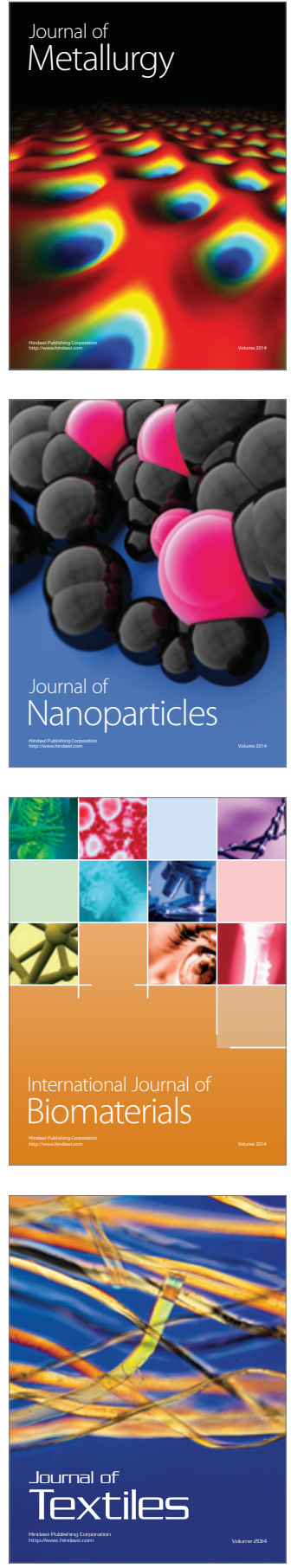